\title{
Pasividad en solución de fosfato de aceros inoxidables solidificados unidireccionalmente
}

\author{
Passivity in phosphate solution \\ of unidirectionally solidified stainless steels.
}

\author{
Rodrigo Elvio Burgos ${ }^{1}$, Florencia Alejandra Bruera ${ }^{1}$, \\ Alicia Esther Ares ${ }^{1}$, Claudia Marcela Méndez ${ }^{1}$
}

${ }^{1}$ UNaM, FCEQyN, Laboratorio de Materiales, Felix de Azara 1554, 3300, Posadas, Misiones, Argentina
Dirección de correo electrónico: cmendez@fceqyn.unam.edu.ar, aares@ fceqyn.unam.edu.ar

\section{RESUMEN}

La pasividad de los aceros inoxidables es atribuida a la formación de una película protectora, conformada por óxidos, sobre la superficie del metal. Esta película posee propiedades semiconductoras y está relacionada con múltiples factores que la hacen de importancia para la comprensión de los fenómenos de corrosión. En este trabajo se estudia la película pasiva formada en aceros inoxidables solidificados unidireccionalmente, $\mathrm{Fe}$ 18Cr-10Ni-2Mo-0,08C (muestra A), Fe-18Cr-14Ni-8Mo-0,03C (muestra C) y Fe-18Cr-10Ni-8Mo-0,08C (muestra F), para determinar la influencia de la variación de la estructura (equiaxial, columnar y con transición de columnar a equiaxial-TCE) en la resistencia a la corrosión, así como investigar la relación entre la resistencia a la corrosión y el espaciamiento dendrítico secundario del material. Para ello se emplean técnicas potenciodinámicas y galvanostáticas, análisis de MottSchottky y espectroscopía de impedancia electroquímica que se realizaron en solución de $\mathrm{Na}_{2} \mathrm{HPO}_{4} 0,5 \mathrm{M}(\mathrm{pH}$ 9,2).Las curvas potenciodinámicas indican una región pasiva y un máximo de corriente en la región transpasiva. Las medidas galvanostáticas realizadas sobre películas pasivas formadas previamente indican dos amesetamientos de potencial, debido al óxido de $\mathrm{Cr}$ y óxido de $\mathrm{Fe}$, del cual surgen dos tiempos de transición. El mayor espesor de óxido de hierro se encuentra en las estructuras equiaxial de la muestra $\mathrm{F}$, que posee el menor espaciamiento dendrítico respecto de los otras dos muestras teniendo en cuenta la misma estructura. El análisis de Mott Schottky muestra que la película pasiva se comporta como un semiconductor tipo $\mathrm{n}$ en el rango de potenciales de $-0,45 \mathrm{~V}$ a $0 \mathrm{~V}$, con una densidad de donores que varía de acuerdo a la composición del acero inoxidable. También se observa que la densidad de donores varía de acuerdo al tipo de estructura para un mismo material.

Palabras Clave: aceros inoxidables, película pasiva, Mott Schottky, resistencia a la corrosión.

\section{ABSTRACT}

Passivity of the stainless steel is attributed to the development of a protector film, formed by oxides on the metal surface. This film has semiconductor properties, and it is important for the understanding of corrosion phenomena.The objective of this work is to study the passive films formed in stainless steels samples solidified unidirectionally. The composition of the alloying elements in the samples are $\mathrm{Fe}-18 \mathrm{Cr}-10 \mathrm{Ni}-2 \mathrm{Mo}-$ 0,08C (sample A), Fe-18Cr-14Ni-8Mo-0,03C (sample C) y Fe-18Cr-10Ni-8Mo-0,08C (sample F), and determine the influence of the structure (equiaxed, columnar and columnar-to-equiaxed transition, CET) in the corrosion resistance, as well as investigate the relationship between the corrosion resistance and the size of the secondary dendritic arm spacing. For this are used potentiodynamic and galvanostatic techniques, Mott Schottky analysis and electrochemical impedance spectroscopy. The measurements were performed in $\mathrm{Na}_{2} \mathrm{HPO}_{4} 0,5 \mathrm{M}(\mathrm{pH}=9,2)$, at room temperature $\left(25^{\circ} \mathrm{C}\right)$. Potentiodynamic measurements indicate a passive region and a maximum current in the transpassive region. The galvanostatic measurements of the passive films indicate two plain of potential, due to chromium oxide and iron oxide, in which two transition times occur. The greater thickness of the iron oxide is in the equiaxed structures of sample $\mathrm{F}$, which has the smallest dendritic spacing with respect to other two samples, given the same structure. Mott Schottky 
analysis shows that the passive film behaves as an n-type semiconductor in the potential range of $-0.45 \mathrm{~V}$ to 0 $\mathrm{V}$, with a density of-donor that varies according to the composition of stainless steel. It is also observed that the density-donor varies according to the type of structure for the same material.

Keywords: stainless steels, passive film, Mott Schottky, corrosion resistance.

\section{INTRODUÇÃO}

En general los aceros inoxidables austeníticos tienen buenas propiedades, entre las cuales se encuentra su excelente resistencia a la corrosión por picado o generalizada en medios clorurados [1]. Por otro lado se sabe que la composición de los aceros inoxidables influye en la macro y microestructura del material, en la formación de inclusiones, y fases secundarias, que a su vez puede dar características particulares a la película pasiva que se forma sobre ellas [2-5]. La película pasiva formada sobre los aceros inoxidables posee propiedades semiconductoras y está relacionada con múltiples factores que la hacen de importancia para la comprensión de los fenómenos de corrosión. El análisis de Mott Schottky es utilizado para obtener información sobre el comportamiento semiconductor de un material de electrodo, en particular el de las películas pasivas formadas anódicamente sobre metales y aleaciones. Se mide la capacitancia como una función del potencial. La capacitancia total, $\mathrm{C}_{\mathrm{T}}$, es generalmente considerada como la contribución en serie tanto de la capa de agotamiento en el espacio de carga en la interfaz película/electrolito, $\mathrm{C}_{\mathrm{SC}}$, y la capa clásica de Helmholtz, $\mathrm{C}_{\mathrm{H}}$. Asumiendo que la capacitancia de la doble capa, $\mathrm{C}_{\mathrm{H}}{ }^{-1}$, es despreciable, la medida de la capacitancia $\mathrm{C}$ es igual a la capacitancia del espacio de carga, $\mathrm{C}_{\mathrm{SC}}$, ecuación (1)

$$
\frac{1}{\mathrm{C}_{\mathrm{SC}}{ }^{2}}=\frac{2}{\varepsilon \varepsilon_{0} \mathrm{eN}_{\mathrm{D}}}\left(E-\mathrm{E}_{\mathrm{bp}}-\frac{\mathrm{kT}}{\mathrm{e}}\right)
$$

donde $\mathrm{N}_{\mathrm{D}}$ es la densidad de especies donoras $\left(\mathrm{cm}^{-3}\right)$, para un semiconductor tipo $\mathrm{n}$ o $\mathrm{p}$, respectivamente, $\varepsilon$ es la constante dieléctrica de la película pasiva $=12[\underline{6}], \varepsilon_{0}=8,85 \times 10^{-14} \mathrm{~F} \mathrm{~cm}^{-1}$ la permitividad del vacío, e $=1,6 \times 10^{-19} \mathrm{C}$ es la carga del electrón, $\mathrm{k}$ la constante de Boltzmann, $\mathrm{T}$ la temperatura y $\mathrm{E}_{\mathrm{bp}}$, el potencial de banda plana.

El cálculo de la capacidad del óxido se realizó suponiendo la aplicabilidad de un circuito equivalente RC serie para describirla respuesta de la impedancia del sistema en la región de más altas frecuencias de medida y fijando $\mathrm{f}=10 \mathrm{kHz}$ donde $\operatorname{Im}(\mathrm{Z})$ es la parte imaginaria de la impedancia medida, ecuación (2).

$$
C=-1 / \omega Z^{\prime \prime}
$$

La cronopotenciometria, permite registrar la evolución temporal del potencial del electrodo conteniendo la película pasivante formadas obre la muestra previamente, y como respuesta a la aplicación de una señal de perturbación en la forma de un escalón de corriente de sentido catódico. Esta respuesta está vinculada con los procesos de electro-reducción que se producen en la interfase película y/o electrodo y el medio electrolítico. Para cada especie que se electrorreduce durante la aplicación del escalón de corriente, el potencial permanece casi invariable (plateau de potencial) mientras la concentración de la especie reaccionante va disminuyendo. Cuando la concentración es suficientemente pequeña, se observa un salto de potencial hacia el valor propio de la electro-reducción de otro sistema redox más difícil de reducir, o finalmente dela reacción de descomposición del electrolito acuoso por electro-reducción. El tiempo transcurrido en la región del plateau de potencial es conocido como tiempo de transición $(\tau)$. Los valores de $\tau$ son directamente proporcional a los espesores de película de óxido a través de la Ley de Faraday [5,7].

Durante el proceso de solidificación unidireccional se obtienen tres tipos de estructuras: columnar, equiaxial, y la zona de transición columnar a equiaxial (TCE), que componen la macroestructura final, siendo el crecimiento dendrítico el proceso más común de cristalización en aceros [8].

El objetivo del trabajo es estudiar las películas pasivas formadas en aceros inoxidables solidificados unidireccionalmente, y determinar la influencia de la variación de la estructura en la resistencia a la corrosión, así como investigar la relación entre la resistencia a la corrosión y el espaciamiento dendrítico secundario del material. Para ello se emplean técnicas potenciodinámicas y galvanostáticas, análisis de MottSchottky y espectroscopía de impedancia electroquímica, todas en solución de $\mathrm{Na}_{2} \mathrm{HPO}_{4}$ que se comporta como buffer, manteniéndose constante el $\mathrm{pH}$ durante las experiencias. La solución seleccionada asegura la formación eficiente de impecables películas pasivas (en ausencia de iones agresivos como el cloruro) [5,7]. 


\section{MATERIALES Y MÉTODOS}

Se trabajó con probetas de acero inoxidable cuya composición se presenta en la Tabla 1, que fueron fundidas y solidificadas en un horno de solidificación horizontal, con extracción de calor desde su base promoviendo la solidificación del material en forma ascendente. Luego de la solidificación direccional se cortaron las probetas en forma longitudinal, se pulieron con lijas de diferentes granulometrías y se atacaron químicamente con solución 1:1:1 de $\mathrm{HCl} / \mathrm{HNO}_{3} / \mathrm{H}_{2} \mathrm{O}$ a temperatura ambiente para revelar la macroestructura. El revelado es necesario para definir las zonas con las cuales se va a trabajar.

Las probetas fueron cortadas transversalmente en cada una de las tres zonas: columnar, equiaxial y con TCE, que luego se lijaron hasta granulometría de CSi \#1200, se pulieron con paño a espejo, se lavaron con agua desmineralizada y alcohol, y se secaron por flujo natural de aire.

Las muestras fueron usadas como electrodos de trabajo en una celda electroquímica de tres electrodos.

Un electrodo de calomel saturado (ECS) fue utilizado como electrodo de referencia, y un electrodo de platino como contra-electrodo.

Tabla1: Composición de las probetas (\%en peso)

\begin{tabular}{|l|l|l|l|l|l|l|l|l|l|}
\hline PROBETA & $\mathbf{C}$ & $\mathbf{S i}$ & $\mathbf{M n}$ & $\mathbf{P}$ & $\mathbf{S}$ & $\mathbf{C r}$ & $\mathbf{N i}$ & $\mathbf{M o}$ & $\mathbf{F e}$ \\
\hline $\mathbf{A}$ & 0,08 & 1,00 & 2,00 & 0,045 & 0,030 & 18,00 & 10,00 & 2,00 & Balance \\
\hline $\mathbf{C}$ & 0,03 & 1,00 & 2,00 & 0,045 & 0,030 & 18,00 & 14,00 & 8,00 & Balance \\
\hline F & 0,08 & 1,00 & 2,00 & 0,045 & 0,030 & 18,00 & 10,00 & 8,00 & Balance \\
\hline
\end{tabular}

Se preparó una solución $0,5 \mathrm{M} \mathrm{Na}_{2} \mathrm{HPO}_{4}, \mathrm{pH}=9,2$, y se trabajó a temperatura entre $25^{\circ} \pm 1^{\circ} \mathrm{C}$.

Previo a cada ensayo, una vez armada la celda, se burbujeó nitrógeno en el seno de la solución electrolítica para remover el oxígeno disuelto, y luego se mantuvo la circulación de nitrógeno por sobre la solución durante la ejecución de cada experimento.

Las medidas potenciodinámicas se realizaron a una velocidad de barrido en sentido anódico de 1 $\mathrm{mV} / \mathrm{s}$. Fueron realizadas por duplicado, obteniendo resultados similares entre ellas.

Se llevaron a cabo reducciones galvanostáticas de las películas anódicas previamente formadas a potencial fijo durante 1hora registrándose los transitorios de potencial resultantes.

El análisis de Mott-Schottky se realizó pasivando anódicamente el electrodo al potencial de formación y aplicándole escalones sucesivos de potencial catódico de $50 \mathrm{mV}$. A cada potencial se midió el espectro de impedancia en una zona de altas frecuencias $\left(10^{5} \mathrm{~Hz}-10^{3} \mathrm{~Hz}\right)$.

\section{RESULTADOS Y DISCUSIÓN}

El revelado de la macroestructura muestra la formación clara de tres regiones bien definidas, equiaxial, transición equiaxial y columnar (Figura 1). En las Figuras 2, 3 y 4 pueden observarse las microestructuras de las muestras.

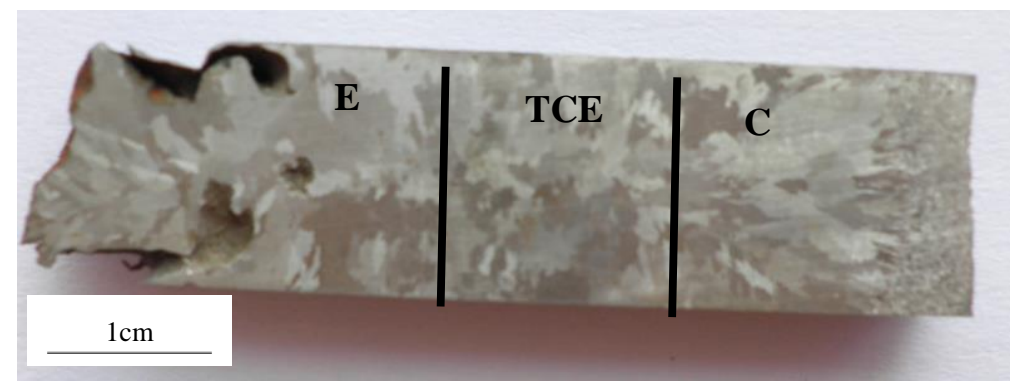

Figura 1: Muestra A obtenida mediante solidificación direccional. 


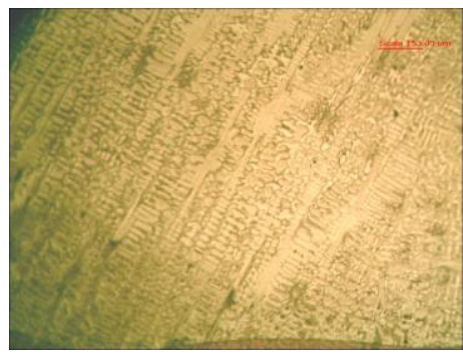

a)

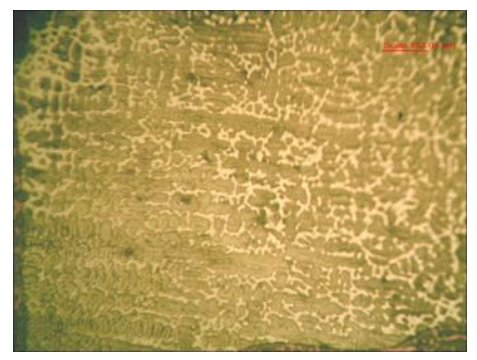

b)

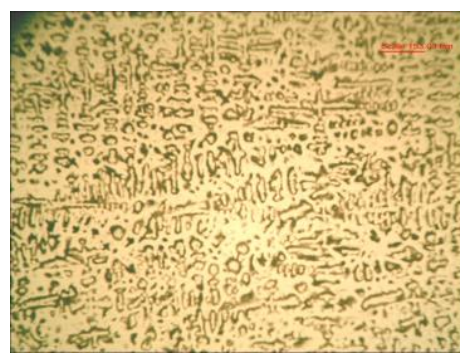

c)

Figura 2: a)Microestructura zona columnar. b) Microestructura zona TCE. c) Microestructura zona equiaxial.

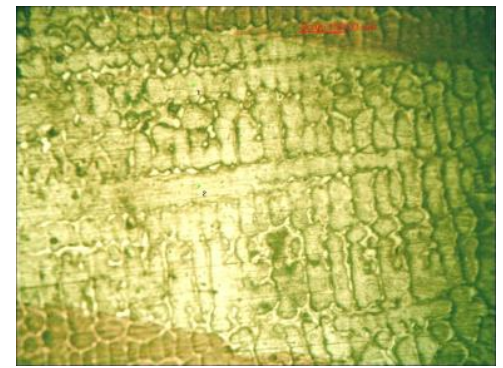

a)

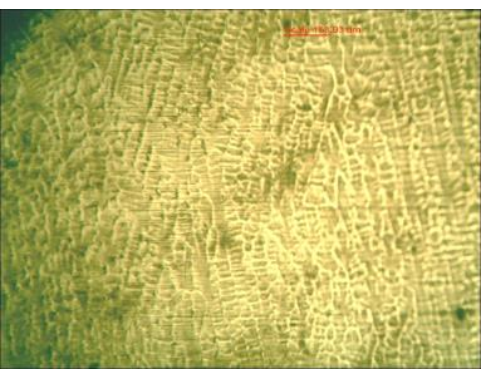

b)

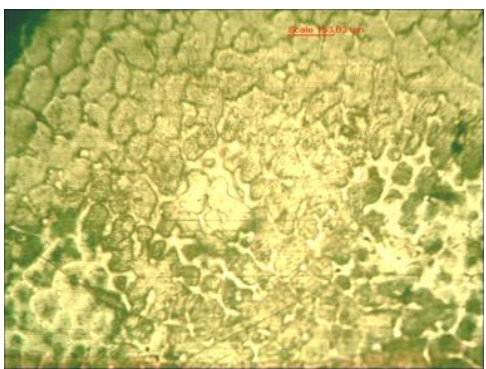

c)

Figura 3: a)Microestructura zona columnar. b) Microestructura zona TCE. c) Microestructura zona equiaxial.

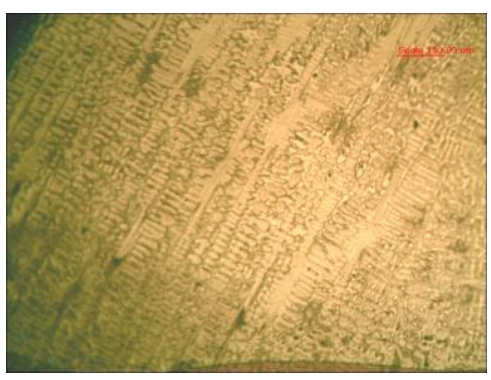

a)

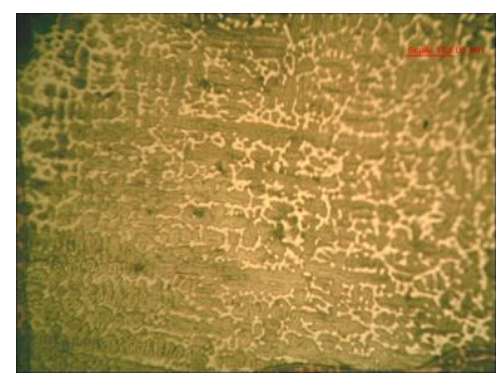

b)

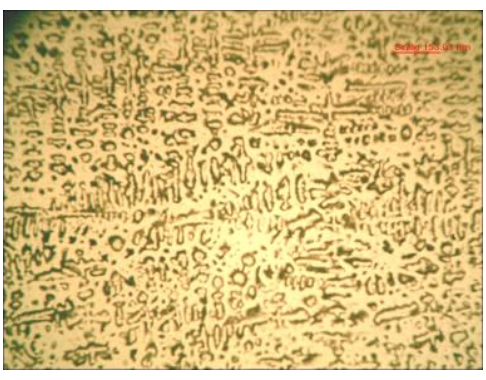

c)

Figura 4: a)Microestructura zona columnar. b) Microestructura zona TCE. c) Microestructura zona equiaxial.

La Figura 4 muestra la relación entre las curvas de polarización potenciodinámicas de las diferentes estructuras del acero. Se observa que para la muestra "A" (Figura 5a), el potencial de corrosión (PC) correspondiente a A3 (estructura columnar) tiene un comportamiento noble relativo a A2 (estructura TCE), y ésta a su vez es noble respecto a A1 (estructura equiaxial). En el caso de "F" (Figura 5c), se observa un comportamiento inverso, siendo F1 más noble, seguido por F2, y luego F3. En las muestras "C" (Figura 5b) la estructura que se muestra con un PC noble es la C1. Los resultados de PC se presentan en la Tabla 2. 


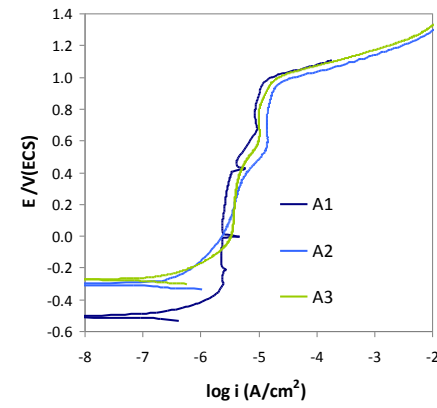

a)

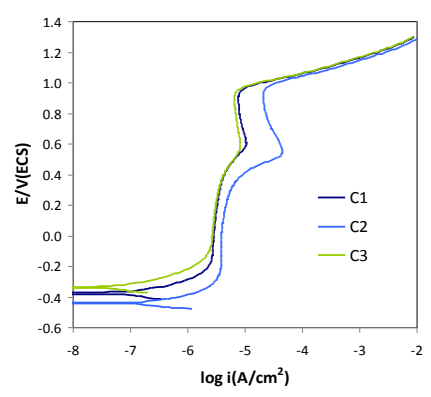

b)

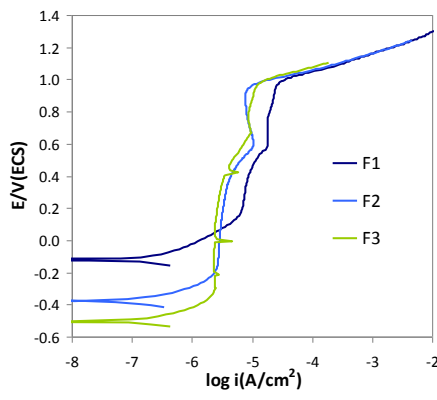

c)

Figura 5: Medidas de polarización potenciodinámica de las muestras A Fe-18Cr-10Ni-2Mo-0,08C(a), C Fe-18Cr-14Ni$8 \mathrm{Mo}-0,03 \mathrm{C}$ (b) y F Fe-18Cr-10Ni-8Mo-0,08C (c)

El desplazamiento de los valores de PC, está vinculado a cambios composicionales y estructurales de la película superficial [9].

Tabla 2: Potenciales de corrosión obtenidos de las curvas potenciodinámicas.

\section{E CORROSIÓN (V)}

\begin{tabular}{l|l|l|l}
\cline { 2 - 4 } & \multicolumn{1}{c|}{ EQUIAXIAL } & \multicolumn{1}{c|}{ TCE } & \multicolumn{1}{c}{ COLUMNAR } \\
\hline $\mathrm{A}$ & $-0,50$ & $-0,30$ & $-0,25$ \\
\hline $\mathrm{F}$ & $-0,10$ & $-0,40$ & $-0,50$ \\
\hline $\mathrm{C}$ & $-0,40$ & $-0,45$ & $-0,35$
\end{tabular}

Las curvas de polarización potenciodinámicas presentan un amplio rango de pasividad y un máximo de corriente en la región transpasiva a un potencial cercano a $0,6 \mathrm{~V}$ asociado a la oxidación $\mathrm{Cr}$ (III), presente en la película pasiva, a $\mathrm{Cr}(\mathrm{VI})$ [10]. En aquellas muestras donde el contenido de Mo es mayor, C y F, se advertiría que el aumento de corriente es mayor, esto podría deberse a que más allá de que el Mo dentro de la región pasiva contribuye beneficiosamente a la misma, cuando llegamos a la región transpasiva la cantidad de $\mathrm{Cr}(\mathrm{VI})$ liberado de las aleaciones aumenta con la adición de Mo [11], si bien la presencia y naturaleza de Mo en la película pasiva es aún profundamente debatido y, por lo tanto, la explicación para el efecto de Mo en la resistencia a la corrosión general de aceros inoxidables es, por el momento, un tema de discusión [12].

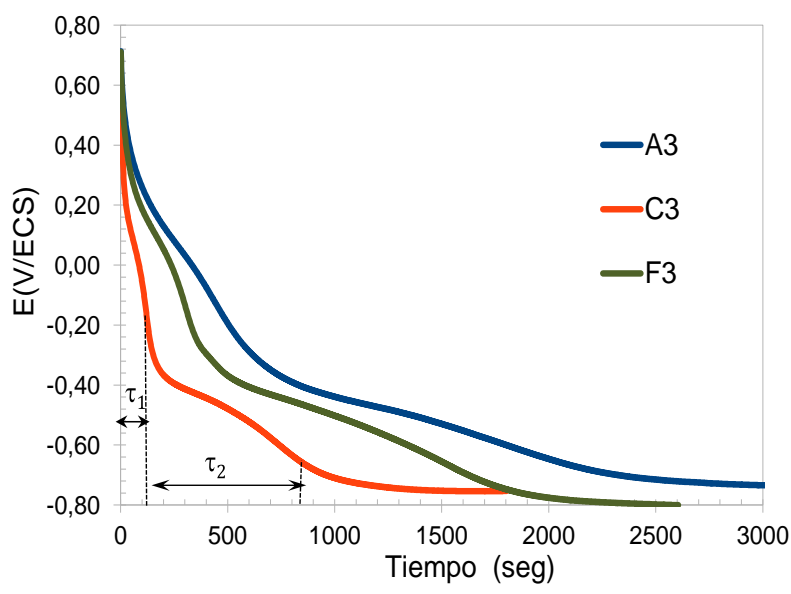

Figura 6: Transitorio de potencial típico de la reducción galvanostática del film anódico de las muestras con estructura columnar. 
La Figura 6 muestra el resultado de la reducción galvanostática, donde se puede observar claramente dos amesetamientos del potencial que definen dos tiempos de transición $\tau_{1}$ y $\tau_{2}$ para procesos de electroreducción. En la Tabla 3, se muestra un resumen de los resultados obtenidos por reducción galvanostática a tres potenciales de formación de película, $\mathrm{E}_{\mathrm{f}}$. Tomando solamente $\tau_{2}$ podemos decir que los mayores espesores lo presentan las estructuras TCE de la muestra A y de la muestra C (excepto al potencial de formación de $300 \mathrm{mV}$ ). Para la muestra F no se puede encontrar una correlación. Para todas las muestras los dos potenciales de electro-reducción se encontraron alrededor de $\mathrm{E}_{1} \approx 0,1 \mathrm{~V}$ y $\mathrm{E}_{2} \approx-0,5 \mathrm{~V}$. Esto indica que se trata de los mismos procesos de electro-reducción independientemente de la composición del metal base.

Las reacciones que podrían llevarse a cabo es la reducción del cromato en solución, con un potencial reversible $\mathrm{E}_{\mathrm{r}}=0.163+0.0197 \log \left[\mathrm{CrO}_{4}{ }^{{ }^{\prime}}\right] \mathrm{V}(\mathrm{ECS})$ al $\mathrm{pH}$ de la solución y que resulta compatible con los valores de $\mathrm{E}_{1}$ asociado a $\tau_{1}$ :

$$
2 \mathrm{CrO}_{4}{ }^{=}+5 \mathrm{H}_{2} \mathrm{O}+6 \mathrm{e}^{-} \Leftrightarrow \mathrm{Cr}_{2} \mathrm{O}_{3}+10 \mathrm{OH}^{-}
$$

A potenciales más catódicos durante $\tau_{2}$ ocurre [13]:

$$
\mathrm{Fe}_{2} \mathrm{O}_{3}+3 \mathrm{H}_{2} \mathrm{O}+4 \mathrm{e}^{-} \Leftrightarrow \mathrm{Fe}^{\mathrm{o}}+\mathrm{Fe}^{++}+6 \mathrm{OH}^{-}
$$

según fuese propuesto en la literatura para amesetamientos de potencial cercanos a $-0,5 \mathrm{~V}$ obtenidos con electrodos de hierro en solución buffer fosfato-borato de $\mathrm{pH}$ comparable al del electrolito utilizado en este trabajo [7].

\begin{tabular}{|c|c|c|c|c|c|c|c|c|c|c|c|c|}
\hline \multicolumn{13}{|c|}{ ESTRUCTURA COLUMNAR } \\
\hline & \multicolumn{4}{|c|}{ MUESTRA A } & \multicolumn{4}{|c|}{ MUESTRA F } & \multicolumn{4}{|c|}{ MUESTRA C } \\
\hline $\mathbf{E}_{\mathrm{f}}(\mathbf{V})$ & $\tau_{1}(\operatorname{seg})$ & $\mathbf{E}_{1}(\mathbf{V})$ & $\tau_{2}(\operatorname{seg})$ & $\mathbf{E}_{2}(\mathbf{V})$ & $\tau_{1}(\operatorname{seg})$ & $\mathbf{E}_{1}(\mathbf{V})$ & $\tau_{2}(\operatorname{seg})$ & $\mathbf{E}_{2}(\mathbf{V})$ & $\tau_{1}(\operatorname{seg})$ & $\mathbf{E}_{1}(\mathbf{V})$ & $\tau_{2}(\operatorname{seg})$ & $\mathbf{E}_{2}(\mathbf{V})$ \\
\hline $\mathbf{0 , 3 0}$ & ---- & ---- & 350 & $-0,40$ & ---- & ---- & 1100 & $-0,50$ & ---- & ---- & 1350 & $-0,55$ \\
\hline $\mathbf{0 , 5 0}$ & ---- & ---- & 550 & $-0,40$ & 150 & 0,0 & 300 & $-0,50$ & 50 & 0,05 & 250 & $-0,50$ \\
\hline $\mathbf{0 , 8 0}$ & 350 & 0,10 & 950 & $-0,50$ & 260 & 0,10 & 1250 & $-0,50$ & 400 & 0,10 & 600 & $-0,40$ \\
\hline \multicolumn{13}{|c|}{ ESTTRUCTURA TRANSICIÓN COLUMNAR EQUIAXIAL } \\
\hline & \multicolumn{4}{|c|}{ MUESTRA A } & \multicolumn{4}{|c|}{ MUESTRA F } & \multicolumn{4}{|c|}{ MUESTRA C } \\
\hline $\mathbf{E}_{\mathbf{f}}(\mathbf{V})$ & $\tau_{1}(\mathrm{seg})$ & $E_{1}(V)$ & $\tau_{2}(\operatorname{seg})$ & $\mathbf{E}_{2}(\mathbf{V})$ & $\tau_{1}(\mathrm{seg})$ & $E_{1}(V)$ & $\tau_{2}(\operatorname{seg})$ & $\mathbf{E}_{2}(\mathbf{V})$ & $\tau_{1}(\mathrm{seg})$ & $E_{1}(V)$ & $\tau_{2}(\mathrm{seg})$ & $\mathbf{E}_{2}(\mathbf{V})$ \\
\hline $\mathbf{0 , 3 0}$ & 50 & 0,0 & 1400 & $-0,50$ & 40 & 0,0 & 600 & $-4,5$ & ---- & $\mid---$ & 520 & $-0,5$ \\
\hline $\mathbf{0 , 5 0}$ & 300 & 0,10 & 1000 & $-0,50$ & 90 & 0,10 & 630 & $-0,50$ & ---- & ---- & 400 & $-0,50$ \\
\hline $\mathbf{0 , 8 0}$ & 500 & 0,10 & 3000 & $-0,50$ & 400 & 0,10 & 1400 & $-0,40$ & 600 & 0,10 & 2600 & $-0,40$ \\
\hline \multicolumn{13}{|c|}{ ESTRUCTURA EQUIAXIAL } \\
\hline & \multicolumn{4}{|c|}{ MUESTRA A } & \multicolumn{4}{|c|}{ MUESTRA F } & \multicolumn{4}{|c|}{ MUESTRA C } \\
\hline $\mathbf{E}_{\mathrm{f}}(\mathbf{V})$ & $\tau_{1}(\mathbf{s e g})$ & $\mathbf{E}_{1}(\mathbf{V})$ & $\tau_{2}(\operatorname{seg})$ & $\mathbf{E}_{2}(\mathbf{V})$ & $\tau_{1}(\mathrm{seg})$ & $\mathbf{E}_{1}(\mathbf{V})$ & $\tau_{2}(\operatorname{seg})$ & $\mathbf{E}_{2}(\mathbf{V})$ & $\tau_{1}(\operatorname{seg})$ & $\mathbf{E}_{1}(\mathbf{V})$ & $\tau_{2}(\operatorname{seg})$ & $\mathbf{E}_{2}(\mathbf{V})$ \\
\hline $\mathbf{0 , 3 0}$ & 60 & 0,0 & 180 & $-0,60$ & 55 & 0,0 & 180 & $-0,50$ & ---- & ---- & 900 & $-0,45$ \\
\hline $\mathbf{0 , 5 0}$ & 60 & 0,10 & 360 & $-0,40$ & 90 & 0,0 & 300 & $-0,40$ & ---- & ---- & 200 & $-0,50$ \\
\hline $\mathbf{0 , 8 0}$ & 480 & 0,10 & 780 & $-0,40$ & 450 & 0,10 & 1850 & $-0,50$ & 60 & 0,10 & 700 & $-0,50$ \\
\hline
\end{tabular}

Tabla 3: Tiempos de transición y potenciales de amesetamiento obtenidos de los experimentos galvanostáticos.

El análisis de MottSchottky (Figura 7) indica un comportamiento lineal con una pendiente característica de óxidos semiconductor tipo $\mathrm{n}$ en el rango de potenciales entre $-0,45 \mathrm{~V}$ a $0,10 \mathrm{~V}$. Los defectos puntuales dentro de la película pasivante pueden ser especies metálicas intersticiales o vacancias de oxígeno, o ambas. Una segunda pendiente puede ser atribuida a una distribución no homogénea de dopaje en los semiconductores, a la presencia de estados de interfaciales o a un segundo estado donor o a una distribución de estados donores sobre el bandgap $[14,15]$. El valor de potencial de banda plana, $\mathrm{E}_{\mathrm{bp}}$, es aproximadamente igual a $-0,45 \mathrm{~V}$, para todas las muestras. 


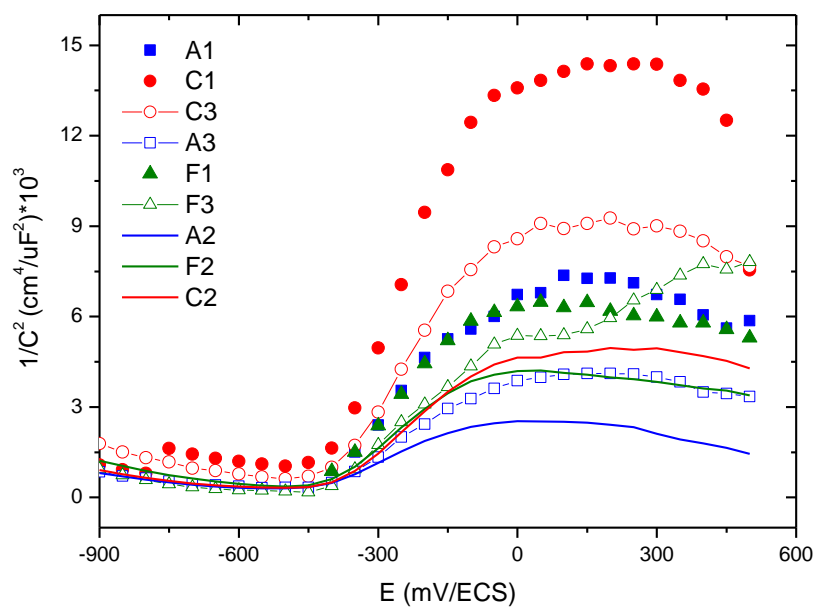

Figura 7: Gráfica de Mott-Schottky para las muestras pasivadas durante 1 hora a partir de C medidas a 10kHz

Los valores de densidad de donores hallados se presentan en la Tabla 4.

Tabla 4: Densidad de donores de películas pasivas.

\begin{tabular}{c|c} 
MUESTRAS & $\mathrm{N}_{\mathrm{D}}\left(10^{20-3}\right)$ \\
\hline $\mathrm{A} 1$ & 8,31 \\
\hline $\mathrm{A} 2$ & 21,7 \\
\hline $\mathrm{A} 3$ & 14,45 \\
\hline $\mathrm{C} 1$ & 4,21 \\
\hline $\mathrm{C} 2$ & 13,16 \\
\hline $\mathrm{C} 3$ & 6,52 \\
\hline $\mathrm{F} 1$ & 8,63 \\
\hline $\mathrm{F} 2$ & 13,27 \\
\hline F3 & 10,43
\end{tabular}

A partir de la Tabla 4 se desprende que la mayor densidad de donores, para cada muestra C, F y A, lo presentan las capas pasivas formadas sobre estructuras de transición columnar - equiaxial, lo que indica una estructura más defectuosa. Los valores entre la muestra A y F son similares tanto para las estructuras columnares y exiaquiales, teniendo en cuenta que la composición entre ellas varían nada más en el contenido de Molibdeno, podemos concluir que la adición del mismo no conduce a una profundo cambio en las propiedades eléctricas y de transporte en las películas de óxido, es decir, no cambia la estructura electrónica de la misma [16]. La diferencia entre la densidad de donores de las estructuras equiaxial y columnar, está asociado a películas más o menos defectuosas, esto se puede relacionar con trabajos anteriores realizados en $\mathrm{NaCl}$ [17], donde las muestras A3 y F3 presentan valores de potencial de picado más bajos respectos a $\mathrm{A} 1$ y $\mathrm{F} 1$, así la estructura columnar (mayor densidad de donores) sufre picado antes que la estructura equiaxial. Si se relacionan las densidades de donores con el espaciamiento dendrítico, obtenido por este mismo grupo de trabajo en publicaciones anteriores [17], se observa que el mayor espaciamiento dendrítico proporciona películas pasivas con menor densidad de donores, en este caso la estructura equiaxial que también presenta una buena capacidad de repasivación tras el picado en medios con cloruros.

La menor densidad de donores en la muestra $\mathrm{C}$, puede deberse al mayor contenido de Níquel en la estructura base [18]. 


\section{CONCLUSIONES}

Los resultados permiten discutir un posible mecanismo de reducción de los óxidos superficiales que involucra solo reacciones de estado sólido. Por otra parte, se pudo confirmar una estructura de la película pasiva comparable a la que fuese informada para otros aceros inoxidables.

La estructura y la conducción electrónica de las películas anódicas formadas sobre los aceros inoxidables se encuentran influenciadas por la composición de las aleaciones y por las diversas macroestructuras que presentan como resultado de la solidificación.

Las vacancias de oxígeno son las especies donoras que determinan un modo de semiconducción electrónica tipo n. Una mayor concentración de defectos puntuales se encuentra relacionado con una estructura más defectuosa de la capa pasivante y una mayor tendencia a la nucleación de picaduras, siendo la densidad de donores, estructura exiaquial < columnar < transición columnar equiaxial. La estructura equiaxial es la que presenta menor concentración de defectos puntuales.

\section{BIBLIOGRAFIA}

[1] PERO-SANZ ELORZ, J.A, Aceros, Metalurgia Física, Selección y Diseño,España, Editoriales Dossat 2000, 2003.

[2] SCHMUKI, P., BÖHNI, H., "Illumination effects on the stability of the passive film on iron", Electrochimica Acta, v.40, pp.775-783, 1995.

[3] GERVASI,C.A., BILMES,P.D., LLORENTE,C.L., Metallurgical factors affecting localized corrosion of low-C 13CrNiMo martensitic stainless steels, chapter 1, New York, I.S. Wang (Ed.), Corrosion Research Trends, Nova Science Publishers, 2007.

[4] BILMES, P.D., LLORENTE, C.L., MENDEZ, C.M., et al.,"Microstructure, heat treatment and pitting corrosion of 13CrNiMo plate and weld metals”, Corrosion Science, v. 51, pp. 876-881, 2009.

[5] GERVASI, C.A., MÉNDEZ, C.M., BILMES, P.D., et al., "Analysis of the impact of alloy microstructural properties on passive films formed on low-C 13CrNiMo martensitic stainless steels", Materials Chemistry and Physics, v.126, pp. 178-182, 2011.

[6] NICIC, I., MACDONALD, D., "The passivity of type 316L stainless steel in borate buffer solution", Journal of Nuclear Materials, v.379, pp.54-58, 2008.

[7] OGURA, K., MAJIMA, T., "Formation and reduction of the passive film on iron in phosphate-borate buffer solution”,Electrochimica Acta, v. 23, pp.1361-1365, 1978.

[8] FLEMINGS, M.C., Solidification Processing, New York, , McGraw Hill Book Co., p. 148, 1947.

[9] VETTER,K.J., Electrochemcial Kinetics: Theoretical and Experimental Aspects, New York , Academic Press, 1967.

[10] KAESCHE,H., Metallic Corrosion, 2nd Edition; Houston TX , NACE, 1985.

[11] BOJINOVA, M., FABRICIUS, G., LAITINEN, T., et al., "Transpassivity mechanism of ironchromium-molybdenum alloys studied by AC impedance, DC resistance and RRDE measurements,"Electrochimica Acta,v. 44, N. 24, pp.4331-4343, 1999.

[12] PARDO, A., MERINO, M.C., COY, A.E., et al., "Effect of Mo and Mn additions on the corrosion behaviour of AISI 304 and 316 stainless steels in H2SO4", Corrosion Science,v 50, pp. 780-794, 2008.

[13] GIACOMELLI,C., GIACOMELLI,F.C., BORTOLLUZZI, R.L., et al., "Properties of potentiostatic passive films grown on iron electrodes immersed in weak - alkaline phosphate solutions", Anti-Corrosion Methods and Materials, v. 53, pp. 232-239, 2006.

[14] GABEN, F., VUILlEMIN, B.,OLTRA,R., "Influence of the Chemical Composition and Electronic Structure of Passive Films Grown on 316L SS on Their Transient Electrochemical Behavior", Journal of The Electrochemical Society, v. 151,n.11, pp. 595-604, 2004.

[15] SCHMUKI,P.,BÖHNI, H., "Metastable pitting and semiconductive properties of passive films", $J$. Electrochemical Society, v. 139, n. 9, pp.1908-1913, 1992.

[16] BETOVAI., BOJINOV, M., ENGLUND, A.,et al."Contact electric impedance and resistance studies of the conduction mechanism in passive films on ferrous alloys", Electrochimica Acta, v. 46, pp. 3627-3640, 2001. 
[17] COVINICH, M.M., MENDEZ,C.M., ARES,A.E., “Comportamiento frente a la corrosión localizada de los aceros inoxidables 316 y316L con diferentes estructuras de solidificación”, in SAM-CONAMET 2013,paperT6C.45, Iguazú, Argentina, 20-23 Agosto 2013.

[18] CHENG, X.Q.; LI, X.G.; DU, C.W.;"Properties of passive film formed on 316L/2205 stainless steel by Mott-Schottky theory and constant current polarization method", Chinese Sci Bull, v. 54, pp.2239-2246, 2009. 